AsCLEPIO. Revista de Historia de la Medicina y de la Ciencia

66 (2), julio-diciembre 2014, p054

ISSN-L:0210-4466

http://dx.doi.org/10.3989/asclepio.2014.18

DOSSIER: BIOPODER Y DETERMINISMOS EN SUDAMÉRICA DURANTE EL SIGLO XX /

DOSSIER: BIOPOWER AND DETERMINISM IN SOUTH AMERICA DURING THE TWENTIETH CENTURY

\title{
EL HUEVO DE LA SERPIENTE AL SUR DEL MUNDO: DESARROLLO Y SUPERVIVENCIA DE LA CIENCIA NAZI EN CHILE (1908-1951)
}

\author{
César Leyton \\ Universidad de Chile \\ cleyton@odontologia.uchile.cl \\ Marcelo Sánchez Delgado \\ Universidad de Chile \\ mjsd.historia@gmail.com
}

Recibido 31 agosto 2013; Aceptado: 14 abril 2014.

Cómo citar este artículo/Citation: Leyton, César y Marcelo Sánchez Delgado (2014), "El huevo de la serpiente al sur del mundo: desarrollo y supervivencia de la ciencia nazi en Chile", Asclepio, 66 (2): p054. doi: http://dx.doi.org/10.3989/asclepio.2014.18

RESUMEN: El trabajo aborda, principalmente a partir de la figura del Dr. Max Westenhoffer y de un trabajo de los Drs. Barrientos y Schirmer de 1937, las conexiones que presenta el desarrollo de la medicina y la antropología en Chile con el pensamiento determinista biológico y racista en Europa. Médicos y antropólogos chilenos como Otto Aichel, Aureliano Oyarzún, Edgardo Schirmer y Juvenal Barrientos dan cuenta de la relación directa de una parte del programa científico chileno de la primera mitad del siglo XX con el programa hereditarista y racista, que alcanzó su punto cúlmine con la eugenesia y la antropología nazi.

PALABRAS CLAVE: Craneometría; Historia de la medicina en Chile; Racismo; Nazismo; Max Westenhoffer.

THE SERPENT'S EGG AT THE SOUTH OF THE WORLD: DEVELOPMENT AND SURVIVAL OF NAZI SCIENCE IN CHILE (1908-1951)

ABSTRACT: The paper presents, mainly through the figure of Dr. Max Westenhoffer and in a work of Drs. Barrientos and Schirmer (1937), the connections between the development of medicine and anthropology in Chile with the development of the biological determinist thought and racism in Europe. Chilean Physicians and anthropologists as Otto Aichel, Aureliano Oyarzún, Edgardo Schirmer and Juvenal Barrientos indicate the direct relationship of the Chilean scientific program of the early twentieth century with the hereditarian and racist program, which reached its climax with the Nazi eugenics.

KEY WORDS: Craniometry; History of Medicine in Chile; Racism; Nazism; Wax Westenhoffer.

Copyright: ( 2014 CSIC. Este es un artículo de acceso abierto distribuido bajo los términos de la licencia Creative Commons Attribution-Non Commercial (by-nc) Spain 3.0. 


\section{INTRODUCCIÓN}

El 12 de Agosto de 1911, en una asamblea popular reunida espontáneamente para hacer homenaje y defensa de la figura del Dr. Max Westenhoffer, el representante de la Escuela Socialista y de la Federación de Zapateros de Santiago, Edmundo Álvarez, se dirigió a los presentes para afirmar que la reunión era «una muestra de civismo y de cultura que quedará grabado (sic) en el corazón de todo hombre sincero, como la bondad sincera que encierra el profesor Sr. Westenhoffer», y más adelante agregaba que "venciendo este gran hombre los convencionalismos de que está poseída la ciencia médica, demostró que en verdad quiere y aprecia a la humanidad toda, demostrando sus conocimientos y criticando la maldad y las causas de ella ${ }^{1}$. Inmerso en un agudo conflicto de competencias profesionales y económicas con los profesores de la Escuela de Medicina de la Universidad de Chile ${ }^{2}$, el médico alemán Max Westenhoffer había presentado su renuncia al cargo de profesor titular de Anatomía Patológica y se encontraba pronto a retornar a su patria. El punto cúlmine del conflicto fue la publicación en 1909 en el Berliner Klinische Wochenschrift de un completo informe de Westenhoffer sobre el estado de la salud pública del país, en el que se comparaba la situación de los enfermos chilenos con la miserable situación sanitaria al interior de África. Los médicos se defendieron corporativamente, mientras los estudiantes y obreros solidarizaron con el sabio alemán, interpretando que este había tomado como suya la bandera de lucha por la dignidad de la atención sanitaria de los marginados. El sabio alemán regresaba a su patria, sin sospechar que la rueda de la fortuna del siglo XX lo traería de vuelta a Chile sucesivamente como eminente anatomopatólogo (1930), como embajador científico del Ministerio de Educación y Culto del Tercer Reich (1938) y en último término, buscando un refugio a su desesperada situación en la Alemania derrotada de 1945.

El científico, que según el representante socialista, guardaba dentro de sí «aprecio a la humanidad toda» publicaría en 1941 en Alemania, en el momento del mayor éxito de la cruzada bélica nacionalsocialista, una obra ciclópea sobre evolución de la especie humana titulada El camino propio evolutivo y el origen del hombre, aplaudida por los científicos del Tercer Reich. La obra mereció un elogioso comentario del Dr. Herbert Fritsche, miembro de la Sociedad Alemana de Ocultismo Científico, en la Revista Mundo Médico de Berlín, quien señalaba que el trabajo de Westenhoffer tenía el mérito de oponerse «al darwinismo utilitario anglosajón» y presentar una teoría evolutiva opuesta a la «teoría universalista de la ausencia de especies y razas», a través de la cual la biología encontraba su conexión "con Linneo y con Goethe». Otros autores que según Fritsche «se ponían positivamente de par- te de la obra de Westenhoffer» (Sievers, 1958, p. 73) eran Arnold Gehlen y Ernst Georg Nauck. Gehlen era sociólogo y filósofo, miembro del partido nazi desde 1933 y Nauck, médico militar y especialista en medicina tropical, que como Director de Higiene durante la ocupación nazi de Varsovia, recomendó la guetización de los judíos polacos. Así, Westenhoffer, el ilustre médico que los obreros y los estudiantes chilenos de 1911 habían defendido en contra de la oligarquía médica, resultó ser, con el paso de los años, una de las figuras notables de la ciencia nacionalsocialista. Embajador cultural del Tercer Reich y figura públicamente homenajeada por el mundo médico alemán en 1942, el nombre de Westenhoffer debía quedar inscrito, para sus apologistas alemanes, en los anales científicos por la teoría de un desarrollo primitivo y particular del hombre, opuesta a su consideración como una especialización de los antropoides (Sievers, 1958, p. 73).

Al terminar la Segunda Guerra Mundial, Westenhoffer se encontró en una precaria situación, «arrinconado en Bondensee (Berlín)» donde "las tropas de ocupación invaden su vivienda y lo reducen a un cuarto miserable» (Sievers, 1958, p. 73). Sin cargos médicos y con una jubilación precaria, fueron sus discípulos chilenos los que acudieron en su auxilio, organizando su regreso al país en 1948, contratado por la Junta Central de Beneficencia. Su obra culmine como biólogo, El camino propio evolutivo y el origen del hombre, será objeto de una nueva edición revisada, la que es publicada en 1951 por la Universidad de Chile, con la autorización de su Rector, Profesor Juvenal Hernández y del Decano de la Facultad de Medicina, Dr. Armando Larraguibel.

La situación de Westenhoffer tras la derrota del nazismo habla por si misma respecto de su compromiso profundo con el Tercer Reich. Dado este contexto ¿Es la publicación de su obra biológica cumbre en Chile en 1951 tan sólo un cúmulo de casualidades o representa una tendencia hegemónica en el campo de las ciencias biomédicas chilenas durante la primera mitad del siglo XX? Este trabajo intentará, a través de diferentes líneas que se vinculan con la figura de Max Westenhoffer, aproximarse al panorama en que esta pregunta puede comenzar ser contestada.

\section{¿UNA MEDICINA PRUSIANIZADA?}

En la década de 1830 y por encargo del Museo de Berlín, Bernardo Philippi - técnico náutico formado en Danzig - recorrió las costas del sur de Chile, específicamente las zonas del interior de Valdivia, Osorno y la isla de Chiloé. El anhelo de hacer productivo y civilizado al país, fortaleciendo la influencia de la civilización europea en un continente bárbaro, confluyó con el sueño de Philippi y se dio comienzo a un plan de colonización alemana del sur chileno. Para 1848, Phili- 
ppi era ya «agente de colonización» nombrado por el gobierno chileno y en 1850 llegaron los primeros colonos alemanes (Collier y Sater, 1998, p. 94). Bernardo Philippi resultó clave para otro acontecimiento de importancia en la historia que nos ocupa: la llegada al país de su hermano Rodolfo Armando Philippi, quien, perseguido en Alemania por sus ideas liberales, se embarcó en Hamburgo en dirección a Chile en el año 1851. Había obtenido el título de médico en 1833 en la Universidad de Berlín y tenía amplia formación en zoología y botánica; sus trabajos sobre moluscos de Sicilia le llevaron a ser condecorado por el Rey Federico II de Prusia. En Chile, la presencia de Philippi fue bien recibida por la elite gobernante. El Presidente de la República lo recibió en audiencia y se le prometió que aquí podría "dedicarse en paz a la industria o a la ciencia» (Márquez de la Plata, 1982, p. 19). Desde un comienzo relativamente humilde como rector del Liceo de la Comunidad Alemana de Valdivia y pasando por misiones científicas oficiales encomendadas por el Gobierno, Rodolfo Armando Philippi se demoró tan sólo tres años en llegar a ocupar cargos de importancia en la elite científica nacional promovida desde el gobierno y su proyecto modernizador. Fue rápidamente nombrado Profesor de Zoología y Botánica en 1853 y Profesor de la Facultad de Ciencias Matemáticas y Físicas en 1854 en la Universidad de Chile. A estos cargos académicos se sumó su nombramiento como Director del Museo Nacional de Historia Natural (Cárdenas, 2003).

Para 1860 Philippi se encontraba en una inmejorable posición en el campo científico y marcando la formación médica a través de dos cátedras que se entendían como la base de la formación científica del médico: la zoología médica y la botánica médica. Rodolfo Philippi dejaría las cátedras que tenía en la Escuela de Medicina en manos de su hijo Federico en 1874, que se había formado en botánica y zoología en la Universidad de Halle, Alemania (Cruz Coke, 1995, p. 507). Federico Philippi dejó la docencia en 1906 y sus cátedras fueron asignadas al doctor Federico Johow, quien se había formado como médico, botánico y biólogo en las Universidades de Berlín y Bonn. En definitiva, puede aseverarse que las cátedras decisivas en la orientación científica de los médicos chilenos estuvieron desde 1853 hasta 1925 en manos de profesores alemanes. Esta línea sucesoria de profesores alemanes fue complementada a partir de 1914 con la llegada del académico italiano Juan Noé Crevani. Si bien Noé provenía de una tradición científica nacional diferente, el programa ideológico subyacente a su quehacer académico era de un carácter tanto o más determinista y hereditarista. Las materias que enseñaba Noé en su programa básico de formación biológica del médico eran: «anatomía comparada, citología, histología, mendelismo, evolución, eugenesia, y enfermedades hereditarias» (Cruz Coke, 1995, p. 503). A la vertiente de científicos alemanes se sumaron a la docencia en la Universidad de Chile, médicos que habían llegado al país para atender las necesidades de la comunidad alemana del sur y que dada la solidez de su formación accedían a la condición de profesores, como es el caso de Germán Schneider, que fue profesor clínica médica (Larraín, 2002, p. 365) y José Juan Brunner, profesor de histología (Orrego Luco, 1953, p. 50).

A estas vías de influencia alemana en la medicina chilena de fines del siglo XIX se sumaron otras fuentes de conexión: la de los hijos de los colonos alemanes del sur de Chile que habiendo estudiado medicina en el país deseaban perfeccionarse en la patria de sus padres y la de una política sostenida por el Estado Chileno a través de la Universidad de Chile, durante el periodo entre 1874 y 1940, que promovía la formación en el extranjero de los médicos chilenos, especialmente en Alemania. Los primeros médicos en ser becados para estudiar en Alemania fueron Vicente Izquierdo, Francisco Puelma Tupper, Carlos Sazié y Máximo Cienfuegos (Cruz Coke, 1995, p. 422). Esta política de transferencia de saberes con Alemania se vio fortalecida a partir del gobierno del Presidente José Manuel Balmaceda, a través de un proyecto más amplio que consideró la modernización del ejército bajo el modelo prusiano y la formación de los profesores chilenos en un Instituto Pedagógico, inspirado en el modelo educativo bismarckiano y llevado adelante por un cuerpo docente de alemanes, entre los que destacan Jorge Enrique Schneider, Hans Steffen, Federico Hanssen, Reinaldo Von Lilienthal, Augusto Tafelmacher, Federico Johow, Federico Albert, Rodolfo Lenz y Alfredo Beutell.

\section{LA CÁTEDRA DE ANATOMÍA PATOLÓGICA Y LA LLEGADA A CHILE DEL PROFESOR WESTENHOFFER}

Desde fines del siglo XIX, la necesidad de actualizar y desarrollar en Chile la Anatomía Patológica puso en primera línea la necesidad de encontrar un profesor específicamente en Alemania. Los médicos chilenos veían como indispensable traer a Chile un científico avezado en la anatomía patológica; es decir, en un método orientado a establecer una correlación entre los diagnósticos hechos en vida al paciente, con las huellas de la enfermedad a nivel celular y microscópico en el cuerpo fallecido. La teoría científica que avalaba este procedimiento, la patología celular, se había desarrollado en Alemania por la figura central de las ciencias médicas alemanas en el siglo XIX, el dr. Rudolf Virchow. En definitiva, Max Westenhoffer llego a Chile como discípulo de Virchow.

La Anatomía Patológica comenzó su desarrollo en Chile con Francisco Puelma Tupper, médico chileno que formó parte de la primera generación en realizar 
estudios en Alemania con el apoyo del Estado chileno y que ejercía la cátedra de histopatología a partir de 1881. Puelma Tupper, que también se había formado con Rudolf Virchow en el Hospital La Charité de Berlín, salió de la cátedra como resultado de una purga política posterior a la Guerra Civil chilena de 1891. Uno de sus sucesores en la cátedra fue Aureliano Oyarzún, quien se había graduado de médico en 1885 y había sido enviado a Alemania, donde permaneció entre 1887 y 1891, estudiando, entre otras cátedras, con Rudolf Virchow. La cátedra de Anatomía Patológica fue asumida por Oyarzún, quien siguió cultivando la enseñanza y la práctica de la anatomía patológica bajo el modelo alemán. En el afán por llevar la práctica de la anatomía patológica a niveles de excelencia científica comparables a los de Alemania, Oyarzún chocó con innumerables inconvenientes culturales y materiales, que lo llevaron a renunciar a la cátedra en 1907. Su renuncia llevó a que el Gobierno de Chile encargara a su Ministro Plenipotenciario en Berlín, Augusto Matte, la contratación de un profesor para la cátedra vacante. Ante las consultas oficiales de Matte a las autoridades imperiales, le fue recomendado el profesor Max Westenhoffer, que a la sazón ejercía como jefe del servicio de autopsias del Hospital Moabit de Berlín, Secretario de la Sociedad Médica de Berlín y Director Redactor del órgano oficial de esta institución, el Semanario Médico de Berlín.

Westenhoffer nació el 9 de Febrero de 1871 en Baviera, era hijo de un profesor de estado. Comenzó sus estudios de medicina en 1890 en la Academia Real Médico Quirúrgica Emperador Federico Guillermo, en la Academia Militar de Medicina. En 1894 leyó su tesis de doctorado en la Universidad Federico Guillermo de Berlín, que trataba sobre la destrucción de tejido cerebral producida por la Sífilis. Según relata su principal biógrafo chileno, Hugo Sievers, fue el propio Rudolf Virchow el que, atraído por el prestigio del joven médico militar, lo llamó a ocupar un puesto vacante de ayudante para su cátedra en La Charité ${ }^{3}$. Con la llegada de Westenhoffer a Chile en 1908, no ocurría algo esencialmente nuevo sino que se reafirmaba la tradición germana en la enseñanza científica en general y en la práctica de la anatomía patológica en particular.

Desde que se produjo su contratación, Westenhoffer emprendió un estudió riguroso de la historia, la geografía, el clima y el sistema político chileno. En la medida en que entró en contacto con la realidad chilena, este interés se centró en lo que Sievers llamó «nuestro complejo racial» (Sievers, 1958, p. 58), realizando viajes de carácter etnológico y antropológico al sur del país y estudiando especialmente al grupo étnico araucano ${ }^{4}$. A su llegada a Chile Westenhoffer fue personalmente recibido por el Presidente de la República, Pedro Montt, y el Ministro de Instrucción Pública, Domingo Amunátegui Solar. La disertación inicial de su cátedra en Chile la realizó 31 de Marzo de 1908 y se tituló Desarrollo histórico de la anatomía patológica y de sus métodos de enseñanza (Sievers, 1958, p. 55).

Los honorarios de Westenhoffer cuadruplicaban los de los profesores de más alto rango en ejercicio en la Facultad de Medicina y Farmacia de la Universidad de Chile. Esta situación de privilegio se fue sumando a otros hechos que enturbiaron su relación con los médicos chilenos. El profesor Westenhoffer, a la época con 37 años de edad, tenía gran celo por su actividad académica y promovió fuertemente la dedicación en jornada completa a la enseñanza y la investigación, lo que fue resistido por los médicos chilenos, para quienes la enseñanza en la Universidad de Chile no era sino una forma más de aumentar su prestigio frente a la clientela privada. A este panorama, se agregó una forma de enfrentamiento que afectaba el prestigio de los médicos chilenos, ya que con mayor frecuencia de lo que ellos deseaban, el riguroso protocolo de autopsia llevado adelante por Westenhoffer «significaba, quiérase o no, una revisión de los diagnósticos clínicos y quirúrgicos» (Sievers, 1958, p. 61).

Si por una parte Westenhoffer sumaba logros, como el de haber conformado ya durante 1908 un completo equipo para el futuro Instituto de Anatomía Patológi$\mathrm{ca}$, el director de la Escuela de Medicina, Luis Barros Borgoño, presentaba reclamos al rector debido a que no se hacían las clases encargadas al sabio alemán. Este clima de beligerancia resultó intensificado por la aparición de dos publicaciones realizadas por Westenhoffer en medios alemanes. Un primer texto, bajo la forma de una carta abierta, titulado «Sobre la Enseñanza de la Anatomía Patológica en la Universidad de Santiago de Chile», publicado el 11 de Enero de 1909 en el Berliner Klinische Wochenschrift, daba cuenta de la precaria situación de su trabajo en Chile. Como esta polémica ya estaba apagándose en el medio nacional, la publicación no recibió mayores comentarios. Pero la segunda publicación de Westenhoffer en el mismo semanario, llevó el ánimo de los médicos chilenos a una confrontación definitiva. En 1911, en los número 23 al 27 del Berliner Klinische Wochenschrift, Westenhoffer realizaba en la práctica un diagnóstico de la situación sanitaria del país, cuya crudeza y realismo, ofendieron la idílica y narcisista imagen que los médicos chilenos tenían de si mismos. Entre muchas otras imágenes que laceraban la autoestima de los médicos e higienistas chilenos, Westenhoffer señalaba que la situación sanitaria de los chilenos de clase humilde «no estaría peor en el interior del África» (Sievers, 1958, p. 64). La situación de Westenhoffer en Chile también se vio afectada por su conexión con el llamado «caso Beckert», un hecho criminal ocurrido en Santiago en 1909 y en el que se vieron involucrados funcionarios de la legación alemana en la capital chilena. 


\section{WESTENHOFFER Y EL CASO BECKERT}

El 5 de febrero de 1909 el edificio de la legación alemana en Santiago de Chile amaneció envuelto en llamas. En medio de los escombros y ante la estupefacción del público santiaguino, apareció un cadáver calcinado. Los rumores comenzaron a circular rápidamente y se instaló sin mayor contrapeso la versión que indicaba que los restos hallados pertenecían a Guillermo Beckert, secretario de la legación, y que el mayordomo en servicio, un militar retirado de baja graduación, de nombre Ezequiel Tapia, había desaparecido de Santiago. Dado que el cadáver encontrado en la legación tenía el anillo perteneciente a Beckert y restos de su vestimenta, la versión de un robo y asesinato, perpetrados por Tapia en la persona de Beckert, se asentó rápidamente. Inesperadamente, un testigo declaró haber visto al secretario Beckert en la madrugada del día de la tragedia. Ante este testimonio el juez de la causa, Sr. Juan Bianchi Tupper, designó una comisión para realizar una nueva autopsia, la que quedó formada por Max Westenhoffer, Aureliano Oyarzún y Otto Aichel quienes se expidieron dando autorización para realizar el funeral del diplomático alemán (Valenzuela Basterrica, 1962, p. 142).

Otto Aichel había nacido en Concepción en 1871, ciudad del sur de Chile, en la que su padre Carl Ludwig Oswald Aichel ejercía funciones diplomáticas como cónsul del imperio alemán y el ejercicio libre de la medicina (Cruz Coke, 1995, p. 546). Otto Aichel estudió ciencias, medicina y filosofía en las universidades Ludwig Maximilians de Munich, la Universidad Friedrich Alexander de Erlangen y la Julius Maximilians de Würzburg. En 1898 recibió su título de médico y al poco tiempo volvió a Chile. En 1902 fue nombrado profesor de ginecología en la Escuela de Medicina de la Universidad de Chile, cátedra que ejerció por poco menos de una década (Cruz Coke, 1995, p. 492).

Más allá de las conspiraciones geopolíticas suscitadas (Délano, 1954, pp. 241-242), y la resolución final del caso con el juez encargando una nueva autopsia al Dr. Germán Valenzuela Basterrica, cuya conclusión fue que el cadáver pertenecía a Ezequiel Tapia evidenciando los errores cometidos por la comisión que le precedió en su tarea ${ }^{5}$, resulta de interés la actuación concertada allí de Westenhoffer, Aichel y Oyarzún. La relación entre Otto Aichel y Aureliano Oyarzún tampoco fue meramente circunstancial a la actuación en la comisión de médicos legistas. Se recordará que el Dr. Oyarzún se había retirado de la Cátedra de Anatomía Patológica, para dedicarse a la antropología. En estas preocupaciones habían coincidido muchas veces $\mathrm{Ai}$ chel y Oyarzún, realizando viajes de investigación arqueológica y antropología racial ${ }^{6}$. Desaprobados por la opinión pública por su actuación en el caso Beckert y con Westenhoffer cargando el peso del escándalo de sus textos considerados difamatorios por el cuerpo médico chileno, Aichel y Westenhoffer emprendieron el regreso a Alemania. Aureliano Oyarzún, por su parte, alegando "razones de salud» parte también a Alemania un poco más tarde, donde permanece hasta 1913 (Orellana Rodríguez, 1979, p. 11).

\section{OTTO AICHEL, UN CHILENO EN LA CIENCIA NAZI}

Al volver a Alemania en 1911, después de su estadía de casi una década en Chile, el país de su nacimiento, Aichel se incorporó al Instituto Anatómico de la Universidad de Halle y en 1914 se trasladó a la Universidad de Kiel. Se incorporó al cuerpo médico del ejército alemán durante la Primera Guerra Mundial y fue jefe médico del hospital militar de Amberes. En la época de la República de Weimar, la carrera científica de Otto Aichel fue en ascenso; se le incorporó a la Academia Alemana de Ciencias y llegó a ser profesor titular de la cátedra de antropología en la Universidad de Kiel. En esa condición, Aichel era parte del programa del Instituto Kaiser Wilhelm de Antropología, Herencia Humana y Eugenesia, "Ciencia de la Raza Alemana», el que llegó a tener decenas de volúmenes publicados en torno a esta materia y que comprendía estudios monográficos sobre determinadas regiones y ciudades alemanas, en relación a temas como fertilidad, medidas craneométricas, antropometría y otros similares. Aichel fue también designado Director del Museo de Antropología de la Universidad de Kiel (Schmuhl, 2008). Desde su posición de privilegio en la Antropología alemana, Aichel continuó los viajes arqueológicos en América del Sur con un énfasis propiamente antropométrico, como queda claro por su publicación de 1932, "Ergebnisse einer Forschungsreise nach Chile-Bolivie (Los resultados de un viaje de investigación a Chile-Bolivia)», un trabajo publicado en la Zeitschrift für Morphologie und Anthropologie y en su texto Der Deutsche Mensch de 1933, realizado, como señalaba el subtítulo de la publicación en base a materiales "europeos y extraeuropeos» (Aichel, 1933).

Su relación con el medio académico chileno, por otra parte, se mantuvo a través de su filiación a la Academia Chilena de Ciencias Naturales. En el número de 1927, la Revista Universitaria de la Universidad Católica de Chile, publicó un opúsculo de Aichel, titulado "La importancia de la herencia en la especie humana» y que por su sólo título habla claramente de la orientación biológica determinista del médico chileno-alemán. Militante del partido nacionalsocialista, Aichel tuvo también una actuación destacada en el proceso de depuración antisemita en la Universidad de Kiel y culminó su carrera dentro de la naciente maquinaria nazi en el gobierno, formando parte de los recién constituidos Tribunales de Salud Hereditaria, que decidían sobre la esterilización obligatoria de los 
«defectuosos»". Aichel fue, en definitiva, una figura de máxima importancia en el programa eugénico alemán. Cuando uno de los más importantes eugenistas brasileños, Renato Kehl emprendió un viaje por Europa para acercase a las principales figuras de la eugenesia en su vertiente anglosajona, su periplo de entrevistas abarcó contactos con Alfred Hermann (Austria), Hermann Lundborg (Suecia), John Alfred Mjoen (Noruega) y los alemanes Hermann Muckermann, Hans Haustein, Eugen Fisher, Vogel Wissenschaftl y Otto Aichel (Souza de, 2007).

\section{AURELIANO OYARZÚN NAVARRO}

Oyarzún se convertiría en la figura fundacional de la antropología y la etnología chilenas, con trabajos que en algunas etapas fueron llevados a cabo, codo a codo, con antropólogos y etnógrafos austríacos (Orellana, 1979). Dedicado desde principios del siglo XX a diversos trabajos arqueológicos, antropológicos y etnológicos, a partir de 1908 su dedicación a estas áreas de investigación es completa. En términos de las escuelas de antropología y etnología que se estaban desarrollando a principios del siglo XX, Oyarzún adscribió a la escuela "histórico cultural» de antropología de Viena, la que a su vez tenía una fuerte influencia del antropólogo Gustav Kossina, filólogo y arqueólogo que dio origen a la teoría de los círculos culturales (Kulturkreise). Esta teoría indicaba que existían «regiones que se identifican con grupos étnicos empleando la dispersión de elementos de cultura material y la difusión cultural, modelo explicativo de la expansión de culturas/pueblos a partir de una "cuna» o lugar de origen» (Ruiz, 1998, p. 148). Las investigaciones y obras de Oyarzún hacia la época del primer centenario republicano de Chile, parecen seguir al pie de la letra este modelo teórico. Específicamente, Oyarzún dedicó en esta época su atención a las influencias materiales y culturales del Imperio Inca y de los Atacameños en la cultura Araucana del cono sur americano. Oyarzún dio a conocer sus estudios sobre la alfarería encontrada en los yacimientos arqueológicos de la costa chilena, en los que identificaba la mítica figura del Trinacrio ${ }^{8}$, un dibujo geométrico presente en la alfarería de los pueblos prehistóricos del Chile central, que Oyarzún hacía remitir a la cultura inca y que interpretó como la representación del mito de la triada (el creador, el sol y el trueno o la tierra, el aire y el agua ${ }^{9}$. Como señala Gonzalo Ruiz, los «círculos culturales» y la "difusión cultural» sirvieron para justificar, en el marco de la arqueología alemana de la década de 1930, la expansión imperial alemana, ya que la arqueología y la paleontología proporcionaban pruebas «de la superioridad militar y racial» (Ruiz, 1998, p.148). Como sabemos, la arqueología y los símbolos de los pueblos de la antigüedad sirvieron de base a la imaginería nazi, llegando al uso institucional de los símbolos solares como la swástica. ¿No era de alguna manera el Trina- crio, en la interpretación de Oyarzún, un símbolo solar del imperialismo incaico, que explicaba a través de los Kulturkreise, las influencias del imperio en las etnias del norte chileno? En definitiva, el trabajo arqueológico de Oyarzún, tiende a replicar la metodología de la arqueología y la antropología alemanas de principios del siglo XX. Cuando en 1937, dos médicos chilenos, inspirados en las enseñanzas de Westenhoffer emprendieron un trabajo antropométrico de dimensiones racistas en enfermos psiquiátricos, manifestaron su profundo agradecimiento a la experticia craneométrica de Aureliano Oyarzún, que les facilitó los materiales y entrenamiento metodológico para las mediciones de cráneos.

\section{MAX WESTENHOFFER EN ALEMANIA: 1911- 1930}

Al volver a Alemania en 1911, Westenhoffer se integró a la actividad clínica y docente, llegando a ser designado, desde Mayo de 1913, profesor de los cursos del Museo Patológico de La Charité en Berlín (Sievers, 1958, p. 70). Tuvo una importante participación como médico militar durante la Primera Guerra Mundial y concluido el conflicto se integró a la burocracia sanitaria de la República de Weimar en diferentes cargos y se jubiló de médico militar en 1929, diez años antes de lo que establecía la ley, prebenda, que según declaraba el mismo Westenhoffer «me fue otorgado para que me pudiera concretar, únicamente, a mis investigaciones que ahora se reducen a la antropología y a la patología comparada» (Sievers, 1958, p. 70). Dada su disponibilidad como anatomopatólogo se produce en este momento su primer regreso a Chile. Westenhoffer fue contratado como Director General de Anatomía Patológica por la Junta de Beneficencia, organización privada que mantenía los hospitales públicos, residiendo en Chile entre 1930 y 1932 . Al volver a Alemania, a fines de 1932, Westenhoffer se dedica a desarrollar y completar los materiales con que llegaría a publicar su obra cumbre sobre evolución, El camino propio evolutivo y el origen del hombre.

Su segundo regreso a Chile se produce el 25 de Septiembre de 1938, arribando al país en comisión científica oficial, junto a los profesores Grossmann de la Universidad de Hamburgo, Volhardt de la Facultad de Medicina de Frankfurt y Huebschmann de la Academia Médica de Hamburgo. La comisión fue recibida por el Presidente de la República y en Santiago se le dio alojamiento en el Club de la Unión, el centro social aristocrático de la capital. Westenhoffer dio una decena de conferencias en distintos puntos del país ${ }^{10}$. De todo su quehacer en esta comisión científica, informó detalladamente a su superior directo, el Ministro de Ciencia, Educación y Cultura del Tercer Reich, Dr. Bernhard Rust, figura fundamental en la purga de los profesores judíos de las universidades alemanas y en la inmersión total de la educación alemana en la doctrina racial del nacionalsocialismo. 
Al volver a Alemania se incorporará al Augusta Hospital de Berlín y de forma muy activa a la Academia Germano-lberoamericana, en el rol de "senador». Esta institución, dirigida por Wilhelm Faupel desde 1934, se había convertido en un activo instrumento de la propaganda nacional socialista para el mundo de habla hispana (Reggiani, 2005). A los 70 años, en medio del apogeo de las victorias de la Wermacht sobre Europa, la revista Mundo Médico le rindió un homenaje (Die medizinische Welt, 1941). La preocupación de los discípulos chilenos por la suerte del maestro alemán al terminar el conflicto bélico con la derrota de Alemania, producen su último y definitivo regreso a Chile en 1948, país al que Westenhoffer consideraba su segunda patria y donde falleció en 1957.

\section{LOS DISCÍPULOS DE 1930}

Entre los discípulos chilenos de Westenhoffer destacó Ernesto Prado Tagle, su primer ayudante médico en la cátedra de Anatomía Patológica de la Escuela de Medicina, iniciada en 1908. Para 1911, cuando Westenhoffer emprendió su primer regreso a Alemania, quedaron dispersos en diferentes hospitales los siguientes médicos anatomopatólogos formados por el sabio alemán: el mismo Ernesto Prado Tagle, Emilio Croizet, Carlos Ugarte, Roberto del Río, Arturo Mardones, Juan de la Vega y Carlos Correa.

Siguiendo la tradición de fines del siglo XIX, el gobierno chileno siguió enviando médicos a perfeccionarse a Alemania hasta los inicios de la segunda guerra mundial. En Enero de 1929 fueron becados para estudiar en el Museo Patológico de La Charité y el Instituto de Anatomía Patológica del Augusta Hospital, donde ejercía Max Westenhoffer, los médicos Ismael Mena, Guillermo Brinck y Teodoro Kausel. Después de la experiencia berlinesa el profesor alemán se hizo cargo de que los becados chilenos siguieran su formación en distintos centros europeos. La relación con estos tres discípulos se profundizaría durante los años de la segunda estadía de Westenhoffer en Chile; es decir, entre 1930 y 1932. En esa oportunidad Westenhoffer puso a estos tres médicos como directores de las unidades de Anatomía Patológica de importantes hospitales y los designó formadores de nuevos ayudantes de la disciplina. El mismo Westenhoffer se hizo cargo del Instituto de Anatomía Patológica del Hospital del Salvador, y allí formó a nuevos discípulos: Juvenal Barrientos, Alberto Guzmán, Eduardo Calderón Paul, Hernán Apablaza, Héctor Rodríguez, Ernestina Pérez, E. Herzog (de la Universidad de Concepción), Pardo Correa, Contreras Stark y Manuel Miranda. Dada la importancia de la formación entregada por Westenhoffer en Anatomía Patológica en los tres periodos en que permaneció en Chile, 1908-1911, 1930-1932 y 1948 hasta su muerte en 1957, puede decirse que casi todos los médicos anatomopatólogos chilenos que ejercieron durante el siglo XX, fueron formados por él o directamente por alguno de sus discípulos.

El Dr. Juvenal Barrientos sería uno de los más activos gestores de la publicación en Chile, en el año 1951, de la obra de evolución biológica por la que Westenhoffer había sido celebrado y aplaudido en Alemania en 1942. El traductor de la edición chilena fue el médico Edgardo Schirmer. La cercanía intelectual e ideológica de Barrientos y Schirmer con la faceta eugenista, evolucionista y antropológica de Westenhoffer tenía antecedentes. Ambos médicos participaron en las Jornadas Neuro-Psiquiátricas Panamericanas, realizadas en Enero de 1937 en Santiago de Chile con un trabajo que hunde sus raíces en la antropología racista y en el determinismo biológico de la primera mitad del siglo XX. Este trabajo tenía un primer antecedente en otro publicado en 1934 en la Revista Médica de Chile por Juvenal Barrientos, en el que, con el fin de aclarar algunos conceptos médicos biológicos sobre el fenómeno de la irritabilidad, había procedido a «esta vulgarización de las ideas del profesor Westenhoffer, según yo las he entendido» (Barrientos Rosas, 1934, p. 360). Dado que el texto de 1937 de Barrientos y Schirmer puede considerarse uno de los puntos de llegada y de cristalización del determinismo biológico de la medicina chilena de la primera mitad del siglo $\mathrm{XX}$, dedicaremos a su estudio el siguiente segmento de este trabajo.

\section{EL TRABAJO DE BARRIENTOS Y SCHIRMER DE 1937: "LA CONSTITUCIÓN DE LA NORMA ANTERIOR DE LA CABEZA ${ }^{11}$}

El trabajo de Barrientos y Schirmer comienza llamando la atención sobre la preferencia que las cuestiones ambientales tenían en medicina a partir del avance de la microbiología; capaz de opacar la tradición de Hipócrates y Galeno en torno a los tipos. Afortunadamente, según Barrientos y Schirmer, «hoy día se ha vuelto al justo término medio, dando igual importancia a los agentes causantes de las enfermedades como al terreno en que evolucionan" (Barrientos y Schirmer, 1938, p. 918). La estructura completa de la metáfora indicaba que el microbio era la semilla y el cuerpo el terreno en que se desarrollaba la enfermedad. El terreno es aquí, por lo tanto, el cuerpo del ser humano ${ }^{12}$

Estas determinantes corporales, el terreno o en términos de la teoría biotipológica de Pende y de Kretschmer "la constitución», conformaban para los autores del trabajo "el cimiento somático vegetativo» sobre el que descansaba "la vida de relación» y por lo tanto la base inevitable para el análisis de la conducta humana. En esta línea de pensamiento, el psicólogo no era sino un fisiólogo especializado; en palabras de Barrientos y Schirmer, «el fisiólogo cúspide» (Ba- 
rrientos y Schirmer, 1938, p. 918). La psicología y la psiquiatría no quedaban entonces dispensadas de la morfología, sino todo lo contrario, profundamente determinadas por ella y por las ciencias que la estudian, la antropometría y la endocrinología. Es el determinismo biológico duro, que iguala fisiología con psicología, lo que Barrientos y Schirmer van a afirmar con su trabajo.

Anclados en esta corriente de pensamiento, los autores advierten que en su trabajo se tratará principalmente de «la constitución morfológica» para «darle mayor importancia y viveza a los signos anatómicos» (Barrientos y Schirmer, 1938, p. 918). La definición de constitución para Barrientos y Schirmer es: «la totalidad de los complejos característicos morfológicos y funcionales, ya heredados (genotipo), ya adquiridos (fenotipo) y cuya base lo constituyen las glándulas de secreción Interna» (Barrientos y Schirmer, 1938, p. 919). Si bien la constitución tiene presencia tanto en lo externo como en lo interno y ambas dimensiones están en relación de correspondencia, el trabajo está dedicado exclusivamente a «los complejos morfológicos externos en la norma anterior de la cabeza» (Barrientos y Schirmer, 1938, p. 919). Para los autores la norma es "el plano en que se observa lo examinado", por lo tanto la norma anterior es la observación que ocurre «cuando se mira el individuo por delante» (Barrientos y Schirmer, 1938, p. 919).

El complejo estudio que emprenderán Barrientos y Schirmer es, en pocas palabras, un estudio de la cara. Ambos médicos hacen un reconocimiento específico a la ayuda técnica recibida en su esfuerzo craneométrico, por parte del Dr. Aureliano Oyarzún, «iniciador de los estudios antropológicos en Chile y actual Director del Museo Histórico Nacional» (Barrientos y Schirmer, 1938, p. 919-920). De lo anterior, resulta clara la formación craneométrica del Dr. Oyarzún y se produce una natural conexión del estudio en cuestión, con las vertientes antropológicas del determinismo y el racismo. $Y$ de paso se puede ver cómo el eugenismo evolucionista de Westenhoffer volvía a juntarse con el de su colega en la comisión médico legal del Caso Beckert, el Dr. Oyarzún.

Por complejos, Barrientos y Schirmer entenderán en definitiva las diferentes presentaciones morfológicas de la cabeza en relación a «tipo, temperamento, raza, edad y sexo" (Barrientos y Schirmer, 1938, p. 919). Reafirmando la determinación biológica y corporal de la conducta, lo autores señalarán que el tipo es la constitución esquelética fija y el temperamento la parte más dinámica y «blanda» de lo corporal (los tejidos, la grasa) y que la relación de estos dos primeros complejos determina «el hábito (del latín habitus, modo de ser)» (Barrientos y Schirmer, 1938, p. 919). Respecto del complejo de la raza, si bien advierten que no es posible hablar de "raza chilena» o "raza aria» (los dos específicos ejemplos que usan los autores), sí puede afirmarse la existencia de "pueblos» $\mathrm{y}$ «lenguas» arias.

El estudio de Barrientos y Schirmer procede a ejemplificar la propuesta con el estudio de la norma anterior de la cabeza en el «enfermo A. B. del Manicomio Nacional de Santiago de Chile, comparativamente en otros enfermos para hacer resaltar más clara y objetivamente las diferencias» (Barrientos y Schirmer, 1938, p. 920). Como quedará claro a través de los diferentes análisis, A.B. es un tipo germánico-ario-craneano, que se pondrá en contraste con tipos mestizos-inferioressubcraneanos. Barrientos y Schirmer usan de forma profusa conceptos técnicos sofisticados de la craneometría alemana, que combinaban el acercamiento geométrico relativo y el acercamiento morfológico objetivo: "vértex» «bregma» «eurión» «tragion», "gonion», "gnation» (Barrientos y Schirmer, 1938, p. 919-923), que pueden medirse con «el compás de tacto" (Barrientos y Schirmer, 1938, p. 921) y que dan origen a diferentes formas de la norma anterior de la cabeza. En lo que respecta al cráneo, los autores distinguen dos formas básicas: las angulosas y las curvas. Las primeras determinan poco desarrollo cerebral, mientras que las segundas determinan formas que los autores consideran propias de los "aristen y eucencefálicos» (Barrientos y Schirmer, 1938, p. 924); es decir, formas de aristocracia y de belleza en las formas del cráneo. En el diagnóstico de A. B., aparece todo el arsenal de medidas y criterios geométricos anatómicos, los que se combinan con conceptos humorales de cuño clásico como el "temperamento seco» (Barrientos y Schirmer, 1938, p. 925).

Las observaciones y nuevos criterios de medición craneométrica llevan a Barrientos y Schirmer a afirmar una regla general que conecta el desarrollo de la cara en desmedro del cráneo, como "propia del hombre primitivo australiano» y del «individuo actual con manifiesto desarrollo de la cara», como evidencian con una foto de un cráneo del Instituto Anatómico Patológico de Santiago de Chile con diagnóstico de «degeneración mental» (Barrientos y Schirmer, 1938, p. 930). La craneometría va estableciendo en forma paulatina la relación entre hombre primitivo, indígenas actuales, degenerados y primates: sobre la configuración "torus supraorbitario» dirán que es "propia del hombre primitivo y del australiano y se acerca al mono» (Barrientos y Schirmer, 1938, p. 931).

La conclusión determinista y craneométrica de Barrientos y Schirmer afirma que la correlación indudable es que «el desarrollo del cráneo está en relación inversa con el desarrollo de la cara», ello por razones "filogenéticas» que entroncan con la posición bípeda estudiada por Westenhoffer en su obra sobre evolución que los autores citan. Todo el repertorio del 
estudio realizado apunta hacia «la importancia de determinar en un individuo humano las medidas que fijan estas diferencias y que tienen gran importancia desde el punto cerebral y mental» (Barrientos y Schirmer, 1938, p. 931). Así, el pensamiento de Barrientos y Schirmer puede verse como una re significación biológica y antropológica de teorías del siglo XIX como la craneología, la frenología y la fisiognomía.

En cuanto los "temperamentos» de la norma anterior de la cabeza, los autores emprenderán un estudio de la distribución de la pilosidad en la cara y cabeza en que mezclan consideraciones endocrinológicas con aspectos temperamentales y morfológicos. Por ejemplo, se designa al primer temperamento «hipertricósico primario, lanuginoso o velloso" (Barrientos y Schirmer, 1938, p. 944). Este enfoque se complementará con estudios y clasificaciones de la epidermis, glándulas sudoríparas, glándulas sebáceas, sistema linfático, sistema circulatorio sanguíneo distribución de las grasas y sistema muscular en su presentación en la cara; siempre relacionando estos sistemas con el aspecto endócrino y clasificaciones temperamentales correspondientes («temperamento seborreico» "temperamento sudoríparo» «temperamento linfático», «temperamento pituitoso», "temperamento exudativo», "temperamento seco», "temperamento hipoplástico vascular», "temperamento gordo», "temperamento flaco ${ }^{13}$ entre muchos otros ${ }^{14}$.

Al analizar el «complejo raza», las precauciones anunciadas en torno al uso del concepto, parecen diluirse de entrada cuando describen que "el vello en el rostro es tanto más desarrollado mientras más inferior es el grupo racial» (Barrientos y Schirmer, 1938, p. 955). La jerarquía de razas en este sentido, para los autores, tiene en el segmento inferior a la raza negra, en un punto intermedio a la raza amarilla y en el superior a la blanca. Por otra parte, el "pelo secundario» que componen cabellos, pestañas y cejas se comporta en forma inversa "es tanto más desarrollado mientras más superior es el grupo racial» (Barrientos y Schirmer, 1938, p. 955). Los autores consideran que el problema racial del vello y del pelo secundario se visibiliza mejor en el cabello de la cabeza y le dedican a este un capítulo de análisis especial en el que desarrollan un detallado análisis de su forma, grosor, tipo de crecimiento, longitud, color, inserción del pelo en el cuero cabelludo y advierten que las características de su herencia se comportan "según las leyes de Mendel» (Barrientos y Schirmer, 1938, p. 958). En la medida en que desarrollan su análisis, la cuestión de las razas se despliega sin contrapeso y siempre en consideración de una polaridad inferior/superior. Al entrar en el análisis del "pelo o terciario o sexual» y realizar la descripción de su distribución por razas, se indica que su ausencia o "distribución discontinua como islotes» es un indicador de «adolescencia racial» (Barrientos y Schirmer, 1938, p. 966), a diferencia de la raza blanca superior cuya distribución es continua. Cabe destacar también que en cuanto al color de los ojos, los autores, después de un detallado esfuerzo descriptivo morfológico, terminan comprendiéndolos como «señales temperamentales o humorales» (Barrientos y Schirmer, 1938, p. 970) y en relación directa con la clasificación racial que traspasa todo el texto. Por su parte, la clasificación de las formas de la nariz considera categorías como la «nariz címbrica o germánica», la "céltica o socrática» la "semita o judía o hitita» (Barrientos y Schirmer, 1938, p. 975), entre otras.

Las mismas cuestiones clasificatorias y descriptivas raciales repiten el mismo alambicado camino descrito hasta aquí en relación a músculos, epidermis, glándulas sebáceas, sudoríparas y otros subsistemas de las "norma anterior de la cabeza»; es decir, de la cara. La cuestión racial, como veremos, tiene un claro objetivo de diagnóstico psiquiátrico y de clasificación;

el objeto de esta esquematización de los caracteres raciales en la norma anterior de la cabeza tiene por objeto orientarnos en la interpretación de ellos, y así poder diagnosticar las mezclas raciales que son las más numerosas, pues las puras son muy raras, y aquellas continuamente se presentan en la práctica (Barrientos y Schirmer, 1938, p. 979).

La "práctica» está constituida en el caso de estos médicos chilenos, por los pacientes del bajo pueblo de Santiago, casi unánimemente formado por mestizos. Los tipos puros son tan excepcionales que ante el hallazgo de un enfermo de raza blanca craneana pura, se ha emprendido el desarrollo del texto, el mencionado "A.B.» del principio del trabajo.

Los complejos analizados en torno a la norma anterior de la cabeza (tipo, temperamento, raza, edad y sexo) remiten a una problemática humoral constitucional. Dado que "la cara es la tarjeta de visita de la constitución general» (Barrientos y Schirmer, 1938, p. 989), el trabajo termina por describir distintos «hábitos»; es decir, lo que los autores entienden por una "manifestación externa del temperamento» (Barrientos y Schirmer, 1938, p. 989). Uno de los aspectos que interesa a los autores del estudio de estos habitus son las formas anómalas que pueda presentar, ya que en ellas se manifiesta "la enfermedad en potencia o latencia intermedia entre lo normal y lo patológico» (Barrientos y Schirmer, 1938, p. 990). Aquí, opera entonces la idea de estigma, ya que es por la «displacia tipológica» - la figura fuera del canon - o por «temperamento dicrásico» (Barrientos y Schirmer, 1938, p. 990) -el comportamiento fuera de la norma- que podrían reconocerse enfermedades latentes. El punto de encuentro de todo el texto y sus casi inconmensurables clasificaciones y subclasificaciones no es otro que la norma del habitus y la capacidad diagnóstica en torno al potencial de enfermedad y peligro. 
En definitiva, los autores conforman un universo tipológico en el que la morfología, la endocrinología, el humorismo y la ciencia del comportamiento se unen para permitir el flujo entre forma y conducta, así como entre forma y morbilidad física y mental. A cada tipo corresponden determinadas enfermedades fisiológicas y mentales. La forma, cuando se sale del canon y la norma, es la enfermedad. El trabajo, viene a ser, parafraseando un texto de Rafael Huertas, un «laboratorio de la norma» (Huertas, 2008). Para su cometido los autores usan con libertad desde la línea eugénica principal norteamericana (Davenport), la tradición alemana (Kretschmer, Westenhoffer), la Biotipología del italiano Pende, entre otras, hasta la tradición humoral precientífica. A modo de ejemplo, el habitus estrictus es: "leptosómico» "cara franca» "hipolástico vascular», "flaco de Davenport», "ectodérmico», "esquizotímico de Kretschmer», "simpaticotónico» "hiposecretorio digestivo, poco bebedor de agua (seco)» "hipotenso», "oligohémico (temperamento frío de Hipocrátes)" (Barrientos y Schirmer, 1938, p. 993-994). La anomalía del tipo remite a: inflamaciones, tumores epiteliales, disturbios de la musculatura lisa y psicosis esquizofrénica, hipertiroidsmo patológico.

En cuanto a los hábitos de las razas, el estudio distingue entre razas primarias y derivadas. Las primarias son la negra, la amarilla y la blanca. La descripción morfológica final de la raza negra remite, aunque los autores no lo dicen explícitamente, al primate y al Neardenthal: «poco frecuente el tipo con predominio craneano», "predominio facial», "ángulo facial agudo», "frente huyente» "potentes mandíbulas», "prognatismo», "boca en forma de hocico». La misma raza negra contiene a su vez cuatro ramas de «evolución cerebral»: hotentotes, papúas, cafres y etíopes. La sentencia por la primitividad y animalidad de la raza negra queda sellada en su siguiente descripción: «En la raza negra predomina la vida afectiva e instintiva sobre el intelecto, observándose en ella el instinto de conservación y sexuales; desde el punto de vista cultural especialmente en las ramas inferiores el lenguaje es en forma de aullidos, guturales, parecido al grito de algunas aves» y, finalmente "la mente del negro es una mente inactiva o pasiva de acuerdo con su morfología cefálica y naturalmente su cultura tan escasa ha sido incapaz de imponerse naturalmente y por lo tanto no ha desempeñado ningún rol civilizador» (Barrientos y Schirmer, 1938, p. 997). En la raza amarilla si bien hay algo más desarrollo cerebral, según admiten los autores, la característica es su «pasividad o pereza», su "vida de predominio mineral o estática» (Barrientos y Schirmer, 1938, p. 997).

La raza blanca es "craneana», con ángulo facial de $90^{\circ}$. Se presenta dividida en xantocroide (rubios) y melanocroide (morenos) y tienen «un predominio franco de la inteligencia y sus culturas han sido expansivas y se han impuesto a las demás razas, es decir, han sido civilizadores». Las lenguas de estas razas se dividen en camitas, semitas o arias o indogermánicas; así las cosas «hasta hoy día las lenguas griegas, aria y latinas, dominan el lenguaje científico internacional» (Barrientos y Schirmer, 1938, p. 998). Las razas secundarias son las que se formaron como combinación de las razas anteriores y se pueden observar, en Chile, «en la clientela hospitalaria (manicomio nacional») (Barrientos y Schirmer, 1938, p. 998). Aquí, en su intento por descubrir enfermedades propias de la «raza chilena» Barrientos y Schirmer vuelven sobre las huellas de Max Westenhoffer, para quien la incidencia de la tuberculosis en Chile tenía un "factor racial» (Barrientos y Schirmer, 1938, p. 998).

\section{A MODO DE CONCLUSIÓN. EL CAMINO PROPIO EVO- LUTIVO Y EL ORIGEN DEL HOMBRE Y LA GÉNESIS DE SU PUBLICACIÓN EN CHILE}

La publicación en Chile de la obra cumbre de las investigaciones sobre biología y evolución de Westenhoffer es producto directo de las gestiones de los discípulos más cercanos al maestro. Fueron los doctores Juvenal Barrientos y Edgardo Schirmer quienes llevaron adelante la tarea. Barrientos ayudó a Westenhoffer con la edición del material y Schirmer se dio a la tarea de traducir del alemán al castellano las más de 500 páginas de una obra de extrema complejidad técnico biológica. Otro discípulo de Westenhoffer, el Dr. Hugo Sievers, hizo gestiones personales para lograr el apoyo financiero del Rector de la Universidad de Chile, Prof. Juvenal Hernández y del Decano de la Facultad de Medicina, Dr. Armando Larraguibel. Todos estos esfuerzos terminaron en la publicación en 1951 por la Editorial Universitaria de la obra que en castellano se llamó El camino propio evolutivo y el origen del hombre.

La obra, se inscribe en la órbita de una ciencia afín al nacionalsocialismo y fue vista con beneplácito por un régimen que si bien hacía una apología burda del darwinismo en todas las dimensiones posibles, se avenía mal con un evolucionismo que hería de manera profunda el narcisismo asociado a una posición relevante del hombre germánico en la naturaleza. La visión evolutiva más apegada a Darwin, en cierto sentido cerraba el camino a las lecturas míticas y racistas de la evolución humana, por su concepto del azar y de la evolución arborescente. Sin entrar en un estudio detallado de esta obra de Westenhoffer aparece claro que se la valoró por reintroducir, esta vez bajo complejos conceptos morfológicos, cierta idealidad de una humanidad original, previa a los grandes primates. Su autor, el anatomopatólogo de mayor importancia en la medicina chilena del siglo XX y uno de los biólogos aclamados por la ciencia del Tercer Reich, 
está enterrado en el Cementerio General de Santiago, en un mausoleo financiado por sus discípulos. La admiración, la filiación discipular y en algunos casos, la afinidad de pensamiento biológico e ideológico quedan manifiestas en ese homenaje, realizado en vida del maestro.

Figuras presentadas en este artículo como las de Federico Johow, Max Westenhoffer, Aureliano Oyarzún, Otto Aichel, Juvenal Barrientos y Edgardo Schirmer Ramos, entre otros, dan cuenta de una línea principal de desarrollo de las ciencias biomédicas en el Chile del siglo XX, cuyo referente principal era la academia alemana, en cuyas fuentes los médicos chilenos bebieron del manantial abundante del determinismo biológico $y$, en el siglo XX, del eugenismo y el racismo más radicalizado. Pudor, ignorancia o estrategia han tendido a ocultar esta vertiente de las ciencias médico antropológicas chilenas. Si por una parte es cierto que los caminos fueron diversos y había espacio a resistencias y apropiaciones diversas ideológicamente, a través de este trabajo hemos querido demostrar que hay una continuidad y armonía, al interior de una parte de la comunidad científico médica chilena de la primera mitad del siglo $\mathrm{XX}$, con las concepciones deterministas biológicas sustentadas al interior de la ciencia nacionalsocialista. En el caso de Barrientos y Schirmer, su línea racista antropológica tenía incidencia directa en su manera de enfrentar el servicio público de psiquiatría en que se desempeñaban, ya que su anhelo era descubrir en la morfología del mestizo los signos de la patología mental correspondiente a su grado de mezcla racial. Así, el artículo de Barrientos y Schirmer representa la cristalización de una tendencia central de las ciencias biomédicas chilenas de la primera mitad del siglo XX. Confluyen en este trabajo la influencia directa de Westenhoffer, el apoyo craneométrico de Oyarzún, el determinismo constitucionalista y una antropología racista en la que se renueva, en términos científicos la posición delirante del "hombre blanco» como único y exclusivo portador de valores, pensamiento, ciencia y civilización.

Aun estando claro que se trataba de publicar en Chile y en 1951, a un autor celebrado por el mundo científico oficial del nazismo, la Universidad de Chile tenía motivos de sobra para dar curso a la edición del libro sobre evolución del Dr. Max Westenhoffer. Era la publicación de uno de los suyos, de una de sus figuras gravitantes en el universo de su Facultad más grande y más querida; era dar continuidad a la gran influencia de la ciencia alemana en la formación del médico chileno de la primera mitad del siglo XX. Por otra parte, la identificación de Westenhoffer con la eugenesia ya eran claras desde la publicación de sus trabajos "Los problemas de la higiene racial» (1920), «Emigración desde el punto de vista eugenésico e Higiene Racial» (1921), «El certificado prematrimonial» (1921) y «Eugenésica y Divorcio» (1927) y queda fuera de toda duda su identificación con el nazismo dado el contexto de su visita al país en 1938. Además, el homenaje y la admiración eran mutuos y públicos. Con motivo del primer centenario de la Universidad de Chile, el 19 de Noviembre de 1942, en el programa "Voz de Alemania» de la Berliner Rundfunk y con el auspicio del Instituto Germano Iberoamericano, Max Westenhoffer hizo un cálido homenaje a la Universidad de Chile. La edición especial del programa culminó con la emisión del tercer acto de Lohengrin de Wagner. Con Europa bajo dominio alemán y en medio de la fatídica campaña nazi en contra de su antiguo aliado soviético, la «solución final» se encontraba muy cercana. La serpiente había salido del huevo. 


\section{NOTAS}

1 Discurso de Edmundo Álvarez en el comicio del 12 de Agosto de 1911 en defensa de Westenhoffer a nombre de la Federación de Estudiantes y de los Estudiantes de Chile. La Mañana. 13 de Agosto de 1911.

2 Al seguimiento de las actividades iniciales de Westenhoffer en Chile (Dávila Boza, 1908), se contrapuso la respuesta corporativa de los médicos chilenos ante la campaña que defendía al patólogo alemán (Sanhueza, 1911).

3 En su segunda estadía en Chile a principios de la década de 1930 Westenhoffer hizo instalar en el Instituto de Anatomía Patológica del Hospital del Salvador en Santiago, el busto de su maestro Rudolf Virchow (Sievers, 1958, p. 54).

4 La edición chilena de su obra sobre evolución El camino propio evolutivo y el origen del hombre contiene fotografías de individuos de la etnia mapuche, las que se adjuntan como documentos de prueba para apoyar la tesis de Westenhoffer en relación a la existencia de un bipedismo humano originario en el desarrollo evolutivo de los vertebrados. La afirmación que querían resumir su posición evolutiva era la de que «el mono desciende del hombre y no el hombre del mono».

5 Días más tarde Guillermo Beckert fue capturado cuando intentaba huir a Argentina por un paso fronterizo en el Sur de Chile. Se le juzgó y fue condenado a muerte. La ejecución ocurrió el 4 de julio de 1910.

6 A fines de la década de 1910, por ejemplo, Oyarzún, Aichel y Von Platten investigaron en conjunto una serie de yacimientos arqueológicos y un sitio funerario en la costa central de Chile (Orellana, 1979, pp. 18-19).

7 Otto Aichel se incorporó al NSDAP el año 1932 y recibió el carnet de miembro № 1273695 .

8 Oyarzún expuso por primera vez sobre el Trinacrio en 1910 en Buenos Aires y sólo un poco más tarde, el 25 de Noviembre de 1911, leyó en la Biblioteca Nacional de Chile su estudio sobre el Trinacrio.

9 Las figuras que hemos observado de este dibujo son sugerentemente parecidas a la swástica.
10 La Revista Médica de Chile anotaba en su sección crónicas que Westenhoffer «desarrolló una intensa labor científica dictando una serie de conferencias en las que divulgó trabajos originales suyos sobre: Antropología, Anatomía Patológica y Comparada, Biología y Medicina». Además, se refiere que Westenhoffer fue declarado miembro honorario de la Facultad de Medicina, la Sociedad Médica de Santiago, de Valparaíso, Sociedad de Tisiología, Sociedad de Anatomía Normal y Patológica y Sociedad de Biología de Concepción, "como una fehaciente prueba de reconocimiento por la labor desarrollada por él entre nosotros y por el impulso que ha dado con sus visitas a la investigación científica del país» (Bénézet, 1938, p. 1086).

11 Ambos médicos se presentan en este trabajo de la siguiente manera: Barrientos como «Prosector del Manicomio Nacional»; es decir como encargado de Anatomía Patológica y Edgardo Schirmer como "Médico del Instituto de Medicina Legal y ayudante del Servicio de Anatomía Patológica del Manicomio de Santiago». En definitiva Barrientos y Schirmer eran colegas en el Servicios de Anatomía Patológica del Manicomio Nacional (Barrientos y Schirmer, 1938, p. 918).

12 Es sabido que la bacteriología y la eugenesia surgieron en forma paralela y en base al mismo paradigma positivista. El binomio "germen y terreno», fue - como documenta un trabajo de Jorge Molero Mesa - un terreno en disputa. Cuanto llevan de ideológico las apropiaciones diferenciadas de ciertas conceptualizaciones médico biológicas queda muy claro al considerar la apropiación anarquista en torno a la dualidad "germen y terreno», la que dio origen a una promoción del naturismo y de un ética biológica, propuesta que Molero Mesa considera una «biopolítica otra» y que se ubica en las antípodas de la lectura de Barrientos y Schirmer en el texto que analizamos (Molero Mesa, 2010).

13 Con seguridad se trata de un sistema clasificatorio del eugenista norteamericano Charles Davenport.

14 Las clasificaciones sucesivas y crípticas de Barrientos y Schirmer, recuerdan el ya famoso fragmento de Borges usado por Foucault en Las palabras y las cosas. 


\section{BIBLIOGRAFÍA}

Aichel, Otto (1933), Der Deutsche Mensch: Studie auf Grund neuen europäischen und aussereuropäischen Material: erste Veröffentlichung der prähistorischen Menschenreste aus Schleswig-Holstein und Beiträge zur Anthropologie Amerikas als Parallele zur europäischen Rassenbildung. Jena, G. Fischer.

Barrientos Rosas, Juvenal (1934), "Concepto de Inflamación como función de adaptación", Revista Médica de Chile, Santiago de Chile, pp. 360-366.

Barrientos, Juvenal y Schirmer, Edgardo (1938), "La constitución de la norma anterior de la cabeza". En: Horwitz, Isaac (ed.), Actas de la Primera Reunión de las Jornadas Neuro-Psiquiátricas Panamericanas, Santiago de Chile, Prensas de Universidad de Chile, pp. 918-999.

Bénézet, Renato (1938), "El Profesor Max Westenhoffer", Revista Médica de Chile, $n^{\circ} 11$, Santiago, p. 1086.

Cárdenas, Mario (2003), "El Mueso Nacional bajo la dirección de Rodulfo A. Philippi 1853-1897", Cuadernos de Historia, $\mathrm{n}^{\circ} 2$, pp. 77-90.

Collier, Simon y Sater, William (1998), Historia de Chile, 1808-1994. Madrid, Cambridge University Press.

Cruz Coke Madrid, Ricardo (1995), Historia de la Medicina Chilena. Santiago de Chile, Editorial Andrés Bello.

Dávila Boza, Ricardo (1908), "Instituto de Anatomía Patológica”, Revista Médica de Chile, n²10, Santiago, pp. 291-295.

Délano, Jorge (1954), Yo soy tu. Santiago, Zigzag Ediciones.

Huertas García-Alejo, Rafael (2008), Los laboratorios de la norma. Medicina y regulación social en el estado liberal. Barcelona, Octaedro-CSIC.

Larraín Aguirre, Camilo (2002), La Sociedad médica de Santiago y el desarrollo histórico de la medicina en Chile. Santiago de Chile, Sociedad Médica de Chile.

Márquez de la Plata, Bernardo (1982), Orígenes del Darwinismo en Chile. Santiago de Chile, Editorial Andrés Bello.
Molero Mesa, Jorge (2010), “Otra manera de ver las cosas. Microbios, eugenesia y ambientalismo radical en el anarquismo español del siglo XX". En: Miranda, Marisa y Gustavo Vallejo (eds.) Derivas de Darwin, Buenos Aires, Siglo XXI.

Orellana Rodríguez, Mario (1979), Compilación, introducción, notas y bibliografía al texto Estudios antropológicos y arqueológicos de Aureliano Oyarzún Navarro. Santiago de Chile, Editorial Universitaria.

Orrego Luco, Augusto (1953), Recuerdos de la Escuela. Santiago de Chile, Editorial del Pacífico.

Reggiani, Andrés (2005), "La ecología institucional de la eugenesia: Repensando las relaciones entre biomedicina y política en la Argentina de entreguerras". En:Miranda, Marisa y Gustavo Vallejo. Darwinismo social y eugenesia en el mundo latino, Buenos Aires, Siglo Veintiuno Editores, pp. 273-310.

Ruiz, Gonzalo (1998), "La distorsión totalitaria: las "raíces prehistóricas" de la España Franquista". En: Huertas, Rafael y Carmen Ortiz (eds.), Ciencia y fascismo, Madrid, Doce Calles, pp. 147-160.

Sanhueza, Ángel (1911), "La Facultad de Medicina y la renuncia del Profesor Westenhoeffer", Revista Médica de Chile, n 9, Santiago, pp. 362-37

Schmuhl, Hans-Walter (2008), The Kaiser Wilhelm Institute for Anthropology, Human Heredity, and Eugenics, 1927-1945. Boston, Springer.

Sievers Wicke, Hugo (1958) "Max Westenhoffer (1871-1957)", Anales de la Universidad de Chile, 112 (4), pp. 47-93.

Souza, Vanderlei de (2007), "La Eugenesia de Renato Kehl y la formación de una red internacional en el periodo entre guerras". En: Miranda, Marisa y Gustavo Vallejo (eds.), Políticas del cuerpo, Buenos Aires, Siglo XXI, pp. 428-429.

Valenzuela Basterrica, Germán (1962), "Informe Médico Legal en el incendio de la legación alemana en Febrero de 1909", Anales Chilenos de Historia de la Medicina, 1 (1), Santiago de Chile, pp. 141-146. 